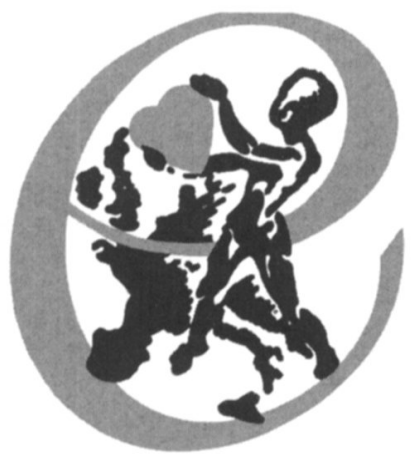

\section{Newsletter from the Association for European Paediatric Cardiology}

\section{The working groups of the Association}

\author{
Ingrid Oberhänsli-Weiss (Secretary-General, Association for European \\ Paediatric Cardiology)
}

$\mathrm{D}$ URING THE LATE 1980S AND THE EARLY 1990s, the Association for European Paediatric Cardiology changed from a club of friends working in the field of paediatric cardiology to a fully structured society. Whilst a club, members had been accepted according to very strict rules. For each University Center, only a single member was accepted, and admission as a full ordinary member was permitted only when he or she had written a thesis and was a recognised paediatric cardiologist. Having become a society, the new structure permitted acceptance of several members from the same center. When they were doctors trained and working as paediatric cardiologists in a European Center, they were welcomed as Ordinary Members. Well known and authoritative paediatric cardiologists from outside of Europe were, and are, welcomed as corresponding members. This strategy self-evidently resulted in the admission of many new members to the society. It is now also the case that colleagues from other medical specialities, when interested in paediatric cardiology and working closely to this field, may similarly become members of the expanded Association.

This growth, which involved not only the Association itself, but also new technical developments, knowledge in paediatric cardiology in general, and the development of interest in special fields within paediatric cardiology, led to the demand for much deeper exchange of experiences in training and skills. In order to meet these new needs, the Association has formed, from 1993 onwards, a series of working groups. This development emulates the longer standing experience of the European Society of Cardiology. The working groups are governed by committees and councils, according to general guidelines which follow very closely the rules of the Association itself, as published on our Web site (www.aepc.org).
Today, in 2002, the Association has 8 working groups, along with a committee governing activities for those concerned with adults or grown-ups with congenital heart disease. These groups are charged with providing specific programmes of teaching in their field, with moving forward the development of their speciality, with creating collaboratiive programmes of research programmes, and with coordinating multicentric studies.

While initially limited to members of the Association, since 1999 the groups have opened their membership. They now accept not only members of the Association who are working more intensively in their particular field, but also those interested in the specific field but without a full training in paediatric cardiology. These colleagues may come from obstetrics, radiology, paediatrics, surgery, embryology, morphology, genetics, and so on. It is no longer necessary to be medically qualified so as to join a working group.

Today, members of any particular group are expected to make an annual contribution of $€ 20$ to the general expenses of their group. This covers mailing, expenses for a lecturer who is not a member of the Association, the promotion of research, teaching sessions, and programmes for study and research.

The current groups are concerned with Arrhythmias and Electrophysiology, Fetal Cardiology, Genetics and Basic Sciences, Imaging, Interventional Cardiology, Morphology, Psycho-Social Problems, and Cardiovascular Surgery, the latter being the most recent group to form. The group concerned with Adults and Grown-ups with Congenital Heart Disease was more recently merged with the corresponding group in the European Society of Cardiology. We now have a committee coordinating these activities in our Association, which has been charged with ensuring continuous contact between ourselves and the European Society of Cardiology. The committee 
organises at least one teaching session during our annual meeting. It is also actively involved in the elaboration and selection of programmes developed within and without the European Society of Cardiology, ensuring active participation in the annual meetings of the Society and its own working group concerned with grown-ups having congenital heart disease.

\section{Teaching activities of the working groups}

While, in the past, the 8 groups provided specific teaching sessions on the day preceding our annual general meeting, it rapidly became obvious that so many sessions in parallel were not appreciated. Many of the participants wanted to follow more than one programme. It was the working group for Fetal Cardiology which initially promoted the change which has occurred over the past 6 years. Now, the teaching sessions incorporate the activities of several working groups. This was achieved by organising sessions for a specific cardiac malformation, with specific aspects covered from fetal to adult life. Each of the groups involved was asked to provide a particular part of the programme, thus producing 6 hours of teaching encompassing morphologic, genetic, embryologic and fetal aspects, new investigatory methods, and surgical and other therapeutic possibilities. These teaching sessions have become very popular, and have attracted many members. Other groups, nonetheless, have continued to organise more specific programmes in their own field, which remain well attended and appreciated. All these programmes have always included presentations of interesting cases, where either diagnostic or therapeutic problems are outlined and discussed in detail.

\section{Continuous medical education}

The activities of the working groups have, from the outset, provided continuous medical education in several specific fields. Our own programme, therefore, predates the recommendations of the European Union of Medical Specialties which now govern such activities.

\section{Courses for teaching and training}

Following the appearance of the recommendations and guidelines from the European Union, and anticipating future regulations to come, the Council of our Association felt obliged to install a specific course for the teaching and training of those responsible for training in paediatric cardiology. It was decided that these courses should be open too for all those members who felt the need for upgrading their knowledge in a specific field. The first of these courses took place in Porto, being concerned with arrhythmias. It filled the day before the official opening of the annual general meeting. Such courses will continue be held in the future on this day prior to the official opening of the annual meeting.

Each year, one of the groups will be asked in alternation to provide the specific programme for teaching and training. For trainees, the course will be provided free of charge. Paediatric cardiologists participating in this course will be asked to pay a small contribution to cover part of the expenses.

It is very much hoped that Industry will help to finance these courses in a generous way. This year's course was generously supported by a teaching grant from Medtronic, while the computers were provided by Hewlett-Packard. This support is greatly appreciated by the Association, which takes this opportunity to express its thanks publicly. The council hopes to improve with such courses the general training in paediatric cardiology, and to promote harmonisation of training programmes within the various European countries.

\section{Scientific meetings}

Scientific sessions organised by the groups in 2002 were fully integrated into the programme of the annual general meeting in Porto. Each working group was allocated two sessions, each of $90 \mathrm{~min}$, covering a specific theme in their sub-specialty, this being over and above the sessions devoted to presentation of abstracts. These important changes in the programme of the annual general meeting resulted, for the first time, in sessions running in parallel. Some sessions remained as plenary presentation, specifically the "State of the Art" lectures and the «Mannheimer» lecture. The programme was much appreciated, acknowledgement being made of the very high scientific standard achieved. Following further reflections, additional changes will probably be introduced for the annual general meeting in 2003, hoping to improve still further the quality and satisfy still more participants.

It must be mentioned that a good number of companies from Industry supported several symposiums organised by groups other than the one concerned with Arrhythmias and Electrophysiology. These sessions were also fully integrated in the scientific programme, and contributed significantly to the high quality already mentioned. We thank again all these generous supporters, hoping that we may count on them also in future years!

\section{Research activities}

Several protocols have already been completed and the results published. Some produced a very high 
rate of participation. Others were less successful in collecting relevant data. In general, it proved difficult to involve many centers in a particular protocol. The small size of most European centers involved with paediatric cardiology, nonetheless, makes it necessary to involve several so as to produce a meaningful study. It is hoped that, in the future, the problems encountered can be managed in a more successful way. E-mail and the Internet will hopefully help to overcome the problems relating to geographical distances, and should serve to increase discussion and promote stimulating interchange. Our web pages can also provide facilities for running a database for combined studies. It will, however, become necessary for very active members of each working group to coordinate the databases, and we hope that Industry will support these studies. The latter support is particularly necessary to overcome logistical problems which can be costly. Until now, Industry has tended to hesitate before supporting studies in children. The need for such studies, nonetheless, is well recognised not only in the European Union, but also in the United States of America. European Commissioner Liikanen has recently addressed this issue in a direct discussion with industry. Thus, cardiovascular drugs have so far not been studied in meaningful fashion in children. The effects, tolerance, metabolism, and side-effects of many drugs are virtually unknown in the paediatric age group. Industry so far does not care to help, as the financial benefit promises to be much less important in children than in adults. The studies, moreover, are more difficult. The Commissioner, nonetheless, emphasised the urgent need for such studies, implying that, if the industry were not willing to support such studies now on a voluntary basis, they might be forced to do so in the very near future. The children of today, of course, are the grown-ups of tomorrow. We have to investigate pharmaceutical therapy in children if we wish to optimise medical treatment and promote the future for the young generations as well as for our own grown-up generation. Many questions in paediatric cardiology need investigation through recruitment of large, and thus meaningful, samples. We are enthusiastic that the Association might become the vehicle to promote such studies. In turn, the council hopes that the individual working groups will establish the questions to be answered, developing as soon as possible protocols to provide a more in-depth look at drug therapy and other new emerging medical developments. Increasingly close collaboration with the corresponding working groups from the European Society of Cardiology should also prove advantageous for future developments.

In summary, the working groups are achieving increasing attention and becoming ever more closely integrated into the everyday activities of our Association. If European collaborative studies are to proliferate, as we hope they will, then to be meaningful and statistically relevant, they will surely require not only the full support of the relevant working group, but also of the Industry. 